Gut, 1988, 29, 1741-1747

Clinical trial

\title{
Balloon or bougie for dilatation of benign oesophageal stricture? An interim report of a randomised controlled trial
}

J G C COX, R K WINTER, S C MASLIN, R JONES, G K BUCKTON, $R$ C HOARE, D $R$ SUTTON, AND J R BENNETT

From the Departments of Gastroenterology and Radiology, Hull Royal Infirmary, Hull and Statistical Services Department, Reckitt and Colman, Hull

SUMMARY Seventy one patients with benign oesophageal strictures were randomised to receive balloon or bougie dilatation. Sixty five patients were eligible for analysis. At the end of five months the balloon group had significantly more dysphagia and the calibre of the strictures in the balloon group had narrowed by a greater degree. The methods were equally safe and acceptable to patients. While the choice of the method of dilatation depends on the individual patient's needs and operator experience, bougie dilatation is more effective in reducing dysphagia and maintaining stricture patency.

Until the early 1980 's, the main dilators in use for dilatation of benign oesophageal strictures at fibreoptic endoscopy in this country were the EderPuestow and Celestin dilators, or their modifications. ${ }^{1}$ Although stricture dilatation using EderPuestow bougies has been practised for over 20 years $^{2}$ there have been few controlled trials comparing the effectiveness of such 'traditional' dilators. ${ }^{3} \mathrm{~A}$ common feature of the dilators is that the forces exerted during dilatation are longitudinal and radial. In 1981 London described the first use of a modern balloon dilator. ${ }^{4}$ Balloons have since gained in popularity, possibly because of the theoretical appeal of applying a localised dilatating pressure in a controlled fashion, exerting only radial force on the stricture. Despite the increasing use of balloon dilators there has been no previous controlled comparison of balloons and traditional bougies.

Address for correspondence: Dr J R Bennett, Department of Gastroenterology, Hull Royal Infirmary, Hull HU3 2 JZ.

Received for publication 21 June 1988.
Methods

PATIENTS

Seventy one patients with benign oesophageal stricture were randomised to receive balloon or bougie dilatation. They were unselected and consecutive, except for patients who lived too far away $(n=2)$ or were too frail to attend for follow up $(n=1)$ and these were excluded from the randomisation. The diagnosis was established in each case by endoscopy (sometimes preceded by radiology) and the benign nature of the condition confirmed by inspection, histology, and cytology. Informed consent to take part in the study was obtained.

Despite careful attempts to exclude patients with malignant strictures, six patients had to be excluded from follow up analysis because of subsequent oesophageal or extrinsic carcinoma (Table 1). Two of these carcinomas were discovered after instrumental perforation had occurred. Thus 65 patients were eligible for analysis: 35 received balloon dilatation and 30 bougie dilatation (Fig. 1). The mean age was 72.3 years in the balloon group and 68.2 years in the bougie group (Table 2 ). The age/sex distribution was 
Table 1 Patients excluded from analysis after randomisation

\begin{tabular}{|c|c|c|}
\hline \multicolumn{2}{|c|}{ Type of stricture Reason excluded } & Course \\
\hline \multicolumn{3}{|l|}{ Balloon group: } \\
\hline 1 Barrett's & Developed malignant ulcer & Oesophagectomy \\
\hline 2 Peptic? & $\begin{array}{l}\text { Perforated, repeat biopsies } \\
\text { malignant }\end{array}$ & Oesophagectomy \\
\hline \multicolumn{3}{|l|}{ Bougie group: } \\
\hline 3 Peptic? & $\begin{array}{l}\text { Progressive course, repeat } \\
\text { biopsies malignant }\end{array}$ & $\begin{array}{l}\text { Died, distant } \\
\text { secondaries }\end{array}$ \\
\hline 4 Peptic? & $\begin{array}{l}\text { Progressive course, infiltrating } \\
\text { carcinoma of bronchus }\end{array}$ & Died \\
\hline 5 Peptic? & $\begin{array}{l}\text { Perforated, repeat biopsies } \\
\text { malignant }\end{array}$ & Died \\
\hline $\begin{array}{l}6 \text { Anastomotic? } \\
\text { (following oe: } \\
\text { for carcinoma }\end{array}$ & $\begin{array}{l}\text { Carcinoma recurred } \\
\text { sophagectomy } \\
\text { a) }\end{array}$ & $\begin{array}{l}\text { Intubation } \\
\text { (failed), XRT, } \\
\text { Died }\end{array}$ \\
\hline
\end{tabular}

Table 2 Randomised patients eligible for analysis

\begin{tabular}{lll}
\hline & Balloon & Bougie \\
\hline Total & 35 & 30 \\
Sex & $23 \mathrm{~F} 12 \mathrm{M}$ & $20 \mathrm{~F} 10 \mathrm{M}$ \\
Mean age & $72 \cdot 3$ years & 68.2 years \\
Age range & $49-93$ years & $30-88$ years \\
Mean stricture diameter & $7 \cdot 60 \mathrm{~mm}$ & $7 \cdot 26 \mathrm{~mm}$ \\
Mean number of previous dilatations & 1.9 & $2 \cdot 5$ \\
\hline
\end{tabular}

Table 3 Aetiology of stricture

\begin{tabular}{lcc}
\hline & Balloon & Bougie \\
\hline Reflux oesophagitis & 26 & 21 \\
Barrett's & 3 & 4 \\
Postoperative: & 3 & 1 \\
$\quad$ reflux surgery & 0 & 2 \\
$\quad$ achalasia surgery & 1 & 0 \\
Postsclerotherapy & 0 & 2 \\
Systemic sclerosis & 1 & 0 \\
Postcricoid & 1 & 0 \\
Caustic & 1 & \\
\hline
\end{tabular}

similar in both groups. The majority of patients had peptic strictures but there was a small number of patients with different aetiologies in each group (Table 3).

The diameter of each stricture was measured using a barium sphere technique developed in this unit. ${ }^{5}$ The spheres are made of high density barium dispersed in witespol $\mathrm{H} 15$ suppository base and range in size from $2 \mathrm{~mm}$ to $16 \mathrm{~mm}$ diameter in $2 \mathrm{~mm}$ increments (Fig. 2). The diameter of the stricture is taken as the diameter of the largest sphere that goes through the stricture. ${ }^{\circ}$

If patients were unable to swallow the barium

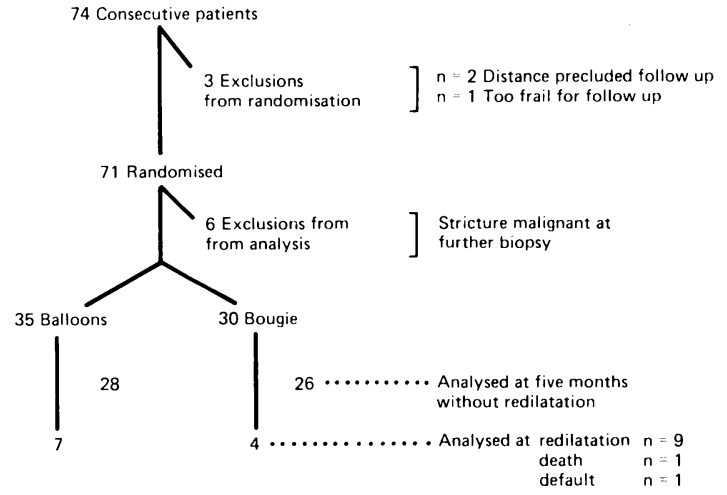

Fig. 1 Outcome of 71 consecutive patients with oesophageal stricture.

spheres, the stricture diameter was measured by a barium swallow technique which has been shown to give reproducible results, though the diameters so measured are somewhat smaller than by spheres. The swallow technique was needed in 10 patients: eight in the balloon group, two in the bougie group. Each patient had the same method for all follow up measurements.

MATERIALS

The Rigiflex oesophageal balloon dilator (TM) (Fig. 3 ) has an $8 \mathrm{~cm}$ long balloon made of polyethylene. The dilator has two lumens, one for the guidewire and one to transmit contrast into the balloon during inflation. The balloon has a diameter uninflated of $3.3 \mathrm{~mm}$ and fully inflated of $20 \mathrm{~mm}$. The maximum diameter of the balloon cannot be exceeded and increasing pressure does not increase the size of the fully inflated balloon. It is designed to tear longitudinally if the burst pressure of $308 \mathrm{kPa}(44 \mathrm{psi})$ is exceeded, so preventing a damaging transverse blowout. The uninflated balloon was passed over a $260 \mathrm{~cm}$ long Teflon coated exchange guide wire under radiological screening. Placement is made easier by tantalum markers proximal and distal to the balloon. The balloon was disinfected in glutaraldehyde before each use.

\section{PROCEDURE}

After randomisation, an assessment of each patient's dysphagia was made using a simple dysphagia score (Table 4) and the stricture was measured. The balloon group strictures were dilated to $20 \mathrm{~mm}(60 \mathrm{~F})$ using radiographic screening to confirm correct placement and full dilatation. The bougie group strictures were dilated in two stages: initially to 18 $\mathrm{mm}(56 \mathrm{~F})$ using Celestin dilators and then dilatation was completed by a Eder-Puestow olive to $19.33 \mathrm{~mm}$ $(58 \mathrm{~F})$, the bougie closest in size to the $20 \mathrm{~mm}$ 


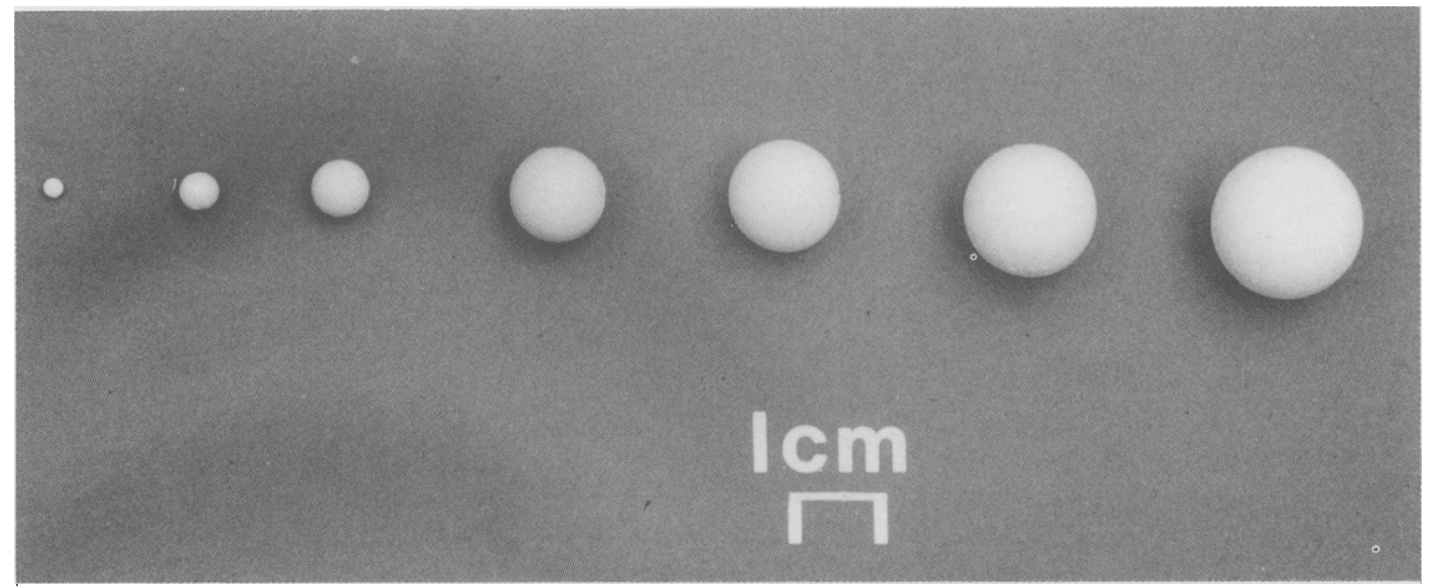

Fig. 2 Barium balls.

balloon. The intention of using the latter combination was to reduce the number of passages of dilators through the mouth, but one patient, known to have a small stomach after surgery, was dilated using bougies only. Radiographic screening was not used for bougienage. All patients in both groups were sedated with intravenous diazepam as Diazemuls (TM) but stronger sedation was given if needed or if past experience had shown this to be necessary for patient comfort. Routine post-dilatation observations of temperature, pulse and blood pressure were done hourly for six hours when, if they were satisfactory, a drink of sterile water was allowed and the frequency of observations reduced. The patients were kept in hospital overnight under observation and allowed home the next day if they ate their breakfast well. (Our patients normally have stayed in overnight after dilatation and although it is now commonplace to allow patients home the same day after dilatation, we included the night's stay in our protocol as at the time the perforation rate with balloons was unknown.) Follow up assessment was initially at one week when a patient acceptability score, dysphagia score, and a repeat stricture measurement were done (Fig. 4). To avoid bias the patient acceptability score was done using a card with the possible alternatives rather than by verbal questioning. The dysphagia score and stricture measurement were repeated at one month post-dilatation and then monthly to five months postdilatation. Patients were exhorted to avoid nonsteroidal anti-inflammatory drugs and cigarette smoking during the period of follow up. Conventional antireflux measures such as raising the head of the bed, antacids, and $\mathrm{H}_{2}$ blockers were advised as necessary. The patients were weighed at each visit.

\section{Results}

The dilatation procedure was accomplished without serious complication in all patients, except the two in whom perforation occurred and whose strictures were subsequently found to be malignant. There was one episode of phlebitis at the injection site of Diazemuls (TM), but this settled spontaneously. Dilatation was incomplete in two balloon patients (initial diameters $8,10 \mathrm{~mm}$ ) and seven bougie patients (initial diameters 4, 4, 4, 6, 8, 10,10 mm). The reasons for incomplete dilatation were the closeness to the burst pressure of the balloon in the

\section{Table 4 Dysphagia score}

\section{Nodysphagia}

Dysphagia with meat

Dysphagia with bread

Dysphagia with semisolids

Dysphagia with liquids

Total dysphagia

Table 5 Patients with redilatations, non-attendance or deaths within five months

\begin{tabular}{lll}
\hline & Balloon & Bougie \\
\hline After one week & 0 & 2 (one redilated, one died of \\
& & pneumonia 4 weeks later) \\
After one month & 2 (redilated) & 0 (redilated) \\
After two months & 4 (redilated) & 1 (rediled to attend) \\
After three months & 1 (redilated) & 1 (failed \\
Total & 7 & 4 \\
\hline
\end{tabular}




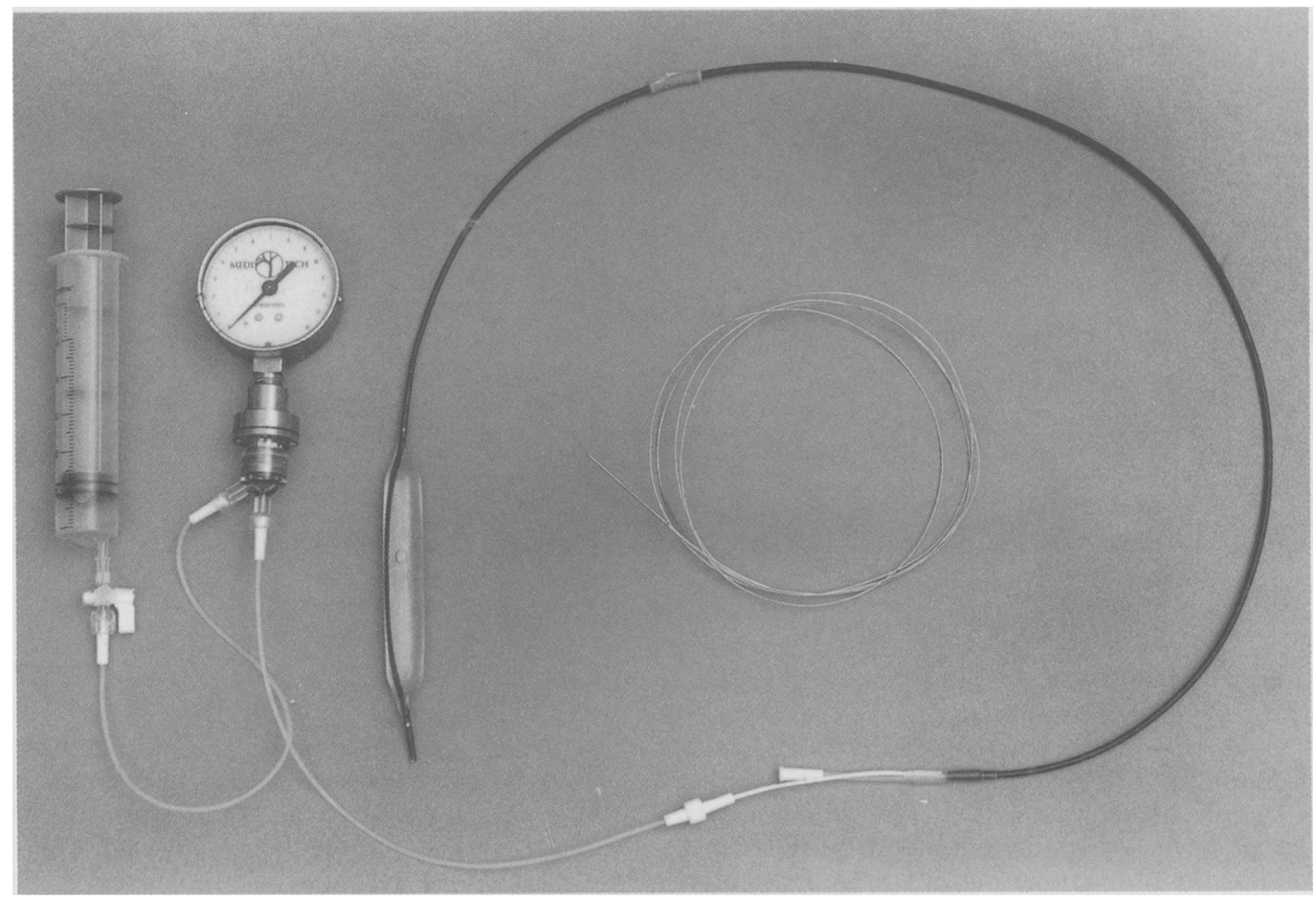

Fig. 3 Rigiflex oesophageal balloon dilator. ${ }^{*}$

balloon group and the 'feel' of excessive force needed to further dilate the stricture in the bougie group.

A further dilatation was offered to all patients if their dysphagia worsened. Repeat dilatation was needed in seven balloon patients and two bougie patients (initial diameters $4,4,4,6,8,8,8 \mathrm{~mm}$ in the balloon group and $4,12 \mathrm{~mm}$ in the bougie group). In all of these patients the previous dilatation had been complete. The difference in the proportions of patients needing redilatation did not reach statistical significance.

Thirty five balloon patients and 30 bougie patients have been followed up to five months post-dilatation, or redilatation or death within the five month period (Table 5). The mean difference from the baseline dysphagia score was significantly better in the bougie group than in the balloon patients at the end of the five month period (balloon mean -0.89, SEM 0.188; bougie mean $-1 \cdot 62$, SEM 0.215: $p=0 \cdot 02$ ) (Fig. 5). Dysphagia scores were analysed using the MannWhitney U-test: the Friedman test for trend over time showed similar results. There was no significant difference in the initial mean stricture diameter in each group. The change in diameters were analysed

*Footnote: The Rigiflex Oesophageal Balloon Dilator (TM) from KeyMed, KeyMed House, Stock Road, Southend-on-Sea, Essex SS2 5QH. from a common baseline level. There was a small difference only between the groups in the mean change from the baseline measurement of stricture

Table 6 Patient acceptability scores

\begin{tabular}{lcc}
\hline & Balloon & Bougic \\
\hline Very unpleasant & 4 & 3 \\
Unpleasant & 2 & 5 \\
Uncomfortable & 4 & 0 \\
Comfortable & 2 & 3 \\
Can't remember it & 23 & 19 \\
\hline
\end{tabular}

No significant difference between groups for patients of all ages or for patients over 75 years.

Table 7 Safety (benign oesophageal strictures only)

\begin{tabular}{|c|c|c|c|}
\hline & & Balloon & Bougie' \\
\hline Perforation & & () & 0 \\
\hline Diazemuls (mg) & & $17 \cdot 72(\mathrm{SE}(0 \cdot 6.52)$ & $17 \cdot 86($ SE 0.789$)$ \\
\hline $\left.\begin{array}{l}\text { Additional } \\
\text { alternative }\end{array}\right\}$ sedation & & 1 & 3 \\
\hline $\begin{array}{l}\text { Screening time for } \\
\text { dilatation ( } \mathrm{min} \text { ) }\end{array}$ & mean & $\begin{array}{l}2 \cdot 3 \\
0 \cdot 75-7\end{array}$ & $\begin{array}{l}0 \\
0\end{array}$ \\
\hline
\end{tabular}




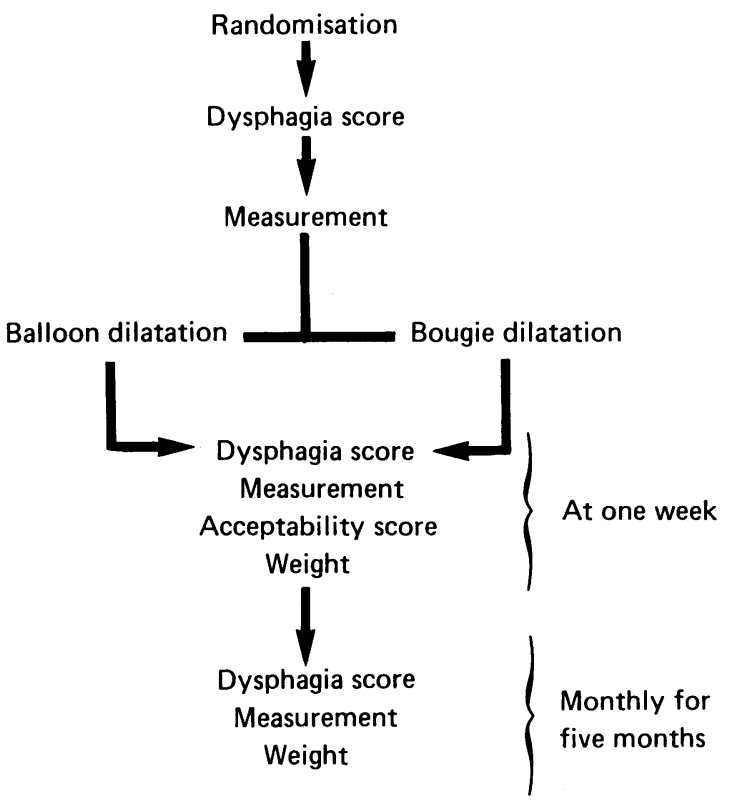

Fig. 4 Procedure after randomisation.

diameter after five months, the strictures in the bougie group remaining wider than the balloon group (balloon mean $1.29 \mathrm{~mm}$, SEM 0.348; bougie mean $2.96 \mathrm{~mm}$, SEM 0.459, $\mathrm{p}=0.06$ ) (Fig. 6). The changes in diameter were analysed using parametric as well as non-parametric methods and similar results were obtained.

Twenty eight balloon patients and 26 bougie patients reached five months post-dilatation without redilatation or death. When these patients were

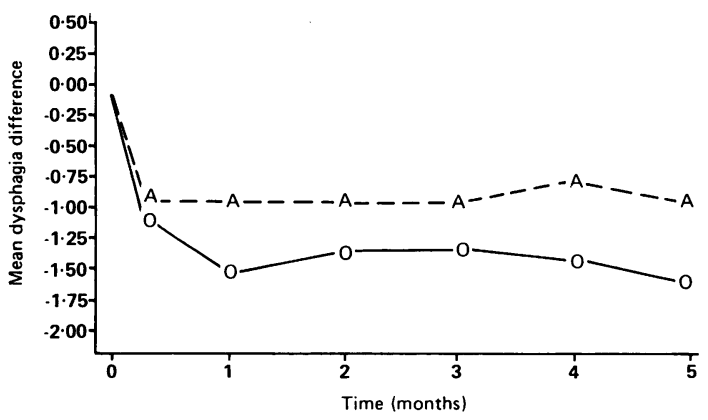

Fig. 5 Dilatation of benign oesophageal stricture: mean difference from baseline dysphagia score against time: $A=$ balloon $O=$ bougie. Patients reaching endpoint -that is, completing five months, redilated or died within five months. Non-parametric analysis for comparison of methods using Mann-Whitney $U$-test on difference in dysphagia score from baseline.

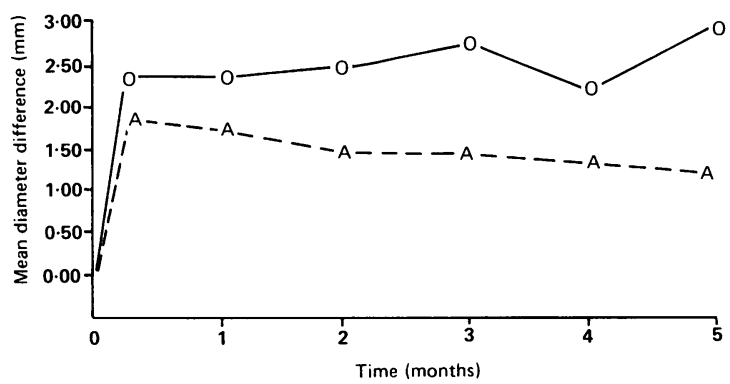

Fig. 6 Dilatation of benign oesophageal stricture: mean difference from baseline stricture diameter against time: $A=$ balloon $O=$ bougie. Patients reaching endpoint - that is, completing five months, redilated or died within five months. Non-parametric analysis of covariants at each time using MRANK procedure of SAS with baseline as covariate.

analysed separately, they again showed a similar difference between the groups in mean change in dysphagia score $(p=0.02)$ and stricture diameter $(p=$ 0.05 ) from baseline measurements. The screening time for patients undergoing balloon dilatation varied from $0.75-7$ minutes, mean 2.3 minutes. Estimating a typical skin dose to be $25 \mathrm{mGy} /$ minute, this leads to a mean incident skin dose of $57.5 \mathrm{mGy}$. There was no statistically significant difference in patients' acceptability scores between the two groups either as a whole or when separately analysed for patients over 75 years of age: Mann-Whitney U-test (Table 6).

The mean amount of Diazemuls (TM) given to each group was similar (one way analysis of variance between methods) (Table 7). One balloon patient and two bougie patients, however, received alternative sedation in the form of intravenous opiates and one bougie patient received intravenous midazolam.

Although more patients in the balloon group lost weight over the five month period (17 balloon patients and six bougie patients lost weight), the maximum weight loss was only $2 \mathrm{~kg}$ in either group. The overall mean weight change was $+0.23 \mathrm{~kg}$ in the balloon group and $+0.57 \mathrm{~kg}$ in the bougie group.

No patient continued with non-steroidal antiinflammatory drugs, but two patients in the bougie group continued with corticosteroids needed for other medical conditions (dermatomyositis and rheumatoid arthritis).

\section{Discussion}

There is a small but clear difference in the effectiveness of the two techniques of dilatation both in terms of symptoms (mean difference from initial dysphagia score) and maintenance of patency of stricture (mean difference from initial stricture size). It is difficult to 
assess the importance of the small numbers in whom measurement was done by barium swallow, or those in whom dilatation was incomplete (although the bougie group did do better than the balloon group despite more incomplete dilatations) or who required repeat dilatation: all these aspects may become clearer as the study progresses.

One of the theoretical advantages of balloon dilators is that the dilating force is applied in a radial direction only in contrast with bougie dilatation which applies an additional longitudinal force on the oesophagus. The implication is that balloons might be safer, although the radial force can be considerable with either technique. ${ }^{7}$ Although no benign stricture was perforated during this study, the rate of perforation with both techniques is small, ${ }^{x 9}$ and a true comparison of safety will be gained when larger numbers of patients are studied. Balloon dilators might seem particularly useful for narrow or tortuous strictures as the narrower tip of the dilator should allow easier negotiation of the stricture. This seemed initially to be true, but as the balloon aged it became stiffer and did not fold so easily for passage through such narrow strictures. The solution may be to store the balloon folded or to use the more pliable transendoscopic balloons (Rigiflex TTS (TM) which also have a smaller uninflated diameter. The narrow flexible Teflon coated guide wire passed easily through difficult strictures: we have not found the need to use a J-tipped wire. We passed the guide wire down a kifa catheter for the study but have since changed to using an amputated nasogastric tube and find that this handles rather better.

A drawback of balloon dilatation is $x$-ray exposure. While the mean incident skin dose of 57.5 $\mathrm{mGy}$ is not excessive (for comparison the average skin dose for a barium meal is $150 \mathrm{mGy}$ ), the need for radiation in what may be a recurrent problem must be carefully assessed. While it is possible to use a balloon dilator without $x$-ray exposure, ${ }^{10}$ the balloon can slip out of the stricture and using screening seems to us far more reliable. Moreover the indentation of the balloon by the stricture not only confirms the position of the balloon but also gives a 'visual feel' in addition to the muted 'tactile feel' through the syringe, adding to the safety of the procedure. The need for $x$-rays could be largely removed by using a transendoscopic balloon (not available at the start of this study), though even these may be easier to place accurately if radiographic screening is used.

The cost of the Rigiflex balloon dilator is $£ 281$, a set of Eder-Puestow dilators $£ 832$ and a pair of Celestin dilators $£ 141$. Balloon life does seem to be limited, although with scrupulous care in handling and cleaning, our balloon has performed over 50 times and another busy department recently performed 90 dilatations before balloon rupture occurred. The practice of using deliberate balloon rupture as an endpoint if a linear balloon profile is not achieved is prohibitively expensive. We never exceeded the burst pressure of the balloon for this reason. We therefore regard monitoring of the inflation pressure as prudent, as if the dilatation is incomplete despite full pressure, the procedure can always be repeated later. The working life of our Eder-Puestow dilator has been over 16 years giving several hundred dilatations; our Celestin dilators have given an average of two years of active use (which is the life span the manufacturing company predict). The Microvasive (TM) pressure manometer used for balloon dilatation costs $£ 345$ but this should have a long working life. Although the Teflon coated guide wires (£35) are more expensive than the steel EderPuestow wires $(£ 20)$, in our hands the former lasted longer, but both need careful handling to ensure maximum use.

Thus we do not at present recommend balloon dilators for routine use in the dilatation of benign oesophageal strictures. They may, however, have a role in special cases such as the following: (1) Patients with particularly narrow or tortuous strictures so long as the balloon has remained supple. (2) Patients who are particularly difficult to endoscope (because the bore of the folded balloon will be less than that of the endoscope). (3) Patients who fear bougie dilatation. Some patients do find the passage of the bougie through the pharynx traumatic despite sedation. We have measured the pressure exerted on the pharynx by bougies and have found it to be sometimes as high as the pressure required for dilatation. Balloons may be less traumatic to the pharynx. (4) Patients with small stomachs in whom a Celestin dilator is contraindicated because of its length (though these patients may be safely dilated by Eder-Puestow dilators). (5) Patients previously perforated in whom a gentle dilatation without longitudinal pressures is desired. (6). Balloons are already in use for children with strictures ${ }^{11-15}$ although no comparative trials have been reported. We have not assessed either technique critically in children but the $x$-ray exposure must be carefully considered ${ }^{16}$ if balloons are to be used, because many of these patients will require repeated procedures.

Our interim conclusions are: (1) There is a statistically significant difference in the change in dysphagia scores five months after dilatation, dysphagia returning more quickly in the balloon group. (2) There is a greater trend towards restenosis in the balloon group. (3) $X$-ray exposure required for a single balloon dilatation is not excessive. (4) Bougie and balloon dilatation appear equally safe as well as equally acceptable to patients. 
We would like to thank Mrs Lynn Brocklesby, Sister Sharon Munro and the nursing staff of the Gastrointestinal Unit, Sister Hazel Daniels and the nursing staff of the Gastrointestinal Ward, Hull Royal Infirmary, Mr G Whitehead of the Department of Medical Illustration, Hull Royal Infirmary, and Mr G Bainbridge, Drs G R Avery, T Griffin, and J F Dyet of the Department of Radiology, Hull Royal Infirmary for their help with this project.

\section{References}

1 Earlham R, Cunha-Melo JR. Benign oesophageal strictures: historical and technical aspects of dilatation. Br J Surg 1981; 68: 829-36.

2 Price JD, Stanciu C, Bennett JR. A safer method of dilating oesophageal strictures. Lancet 1974; 1: 1141-2.

3 Hine KR, Hawkey CJ, Atkinson M, Holmes GKT. Comparison of the Eder-Puestow and Celestin techniques for dilating benign oesophageal strictures. Gut 1984; 25: 1100-2.

4 London RL, Trotman BW, DiMarino AJ, et al. Dilatation of severe oesophageal strictures by an inflatable balloon catheter. Gastroenterology 1981; 80: 173-5.

5 Dyet JF, Bennett JR, Buckton GK, Ashworth D. The radiological measurement of oesophageal stricture diameter. Clin Radiol 1983; 34: 647-9.

6 Bennett JR, Sutton DR, Price JF, Dyet JF. Effects of bougie dilatation on oesophageal stricture size. In: DeMeester TR, Skinner DB, eds. Oesophageal disorders: pathophysiology and therapy. New York: Raven Press, 1985: 221-4.
7 Cox JGC, Buckton GK. Bennett JR. Radial pressures during stricture dilatation with bougies and balloons [Abstract]. Gut 1987; 28: A1370-1.

8 Tulman AB, Worth Boyce H. Complications of oesophageal dilation and guidelines for their prevention. Gastrointest Endosc 1981; 27: 229-34.

9 Kozarek RA. Hydrostatic balloon dilation of gastrointestinal stenoses: a national survey. Gastrointest Endosc 1986; 32: 15-9.

10 Graham DY, Lacey Smith J. Balloon dilatation of benign and malignant oesophageal strictures - blind retrograde balloon dilatation. Gastrointest Endosc 1985; 31: $171-4$.

11 Ball WS, Strife JL, Rosenkrantz J, Towbin RB, Noseworthy J. Oesophageal strictures in children treatment by balloon dilatation. Radiology 1984; 150: 263-4.

12 Goldthorn JF, Ball WS, Wilkinson LG, Seigel RS, Kosloske AM. Oesophageal strictures in children: treatment by serial balloon catheter dilatation. Radiology 1984; 153: 655-8.

13 Stringer DA, Pablot SM, Mancer K. Gruntzig angioplasty dilatation of an oesophageal stricture in an infant. Pediatr Radiol 1985; 15: 424-6.

14 Johnsen A, Ingemann Jensen L, Mauritzen K. Balloon dilatation of oesophageal strictures in children. Pediatr Radiol 1986; 16: 388-91.

15 Sato Y, Frey EE, Smith WL, Pringle KC, Soper RT, Franken EA. Balloon dilatation of oesophageal stenosis in children. AJR 1988; 150: 639-42.

16 Daword RM, Hall C. Too much radiation for too many children? Br Med J 1988; 296: 1277-8. 